\title{
Computational Neurogenetic Modelling: Gene Networks within Neural Networks
}

\author{
Nikola Kasabov, Lubica Benuskova and Simei Gomes Wysoski \\ Knowledge Engineering \& Discovery Research Institute \\ Auckland University of Technology \\ Auckland, New Zealand \\ E-mail: \{nkasabov, lbenusko, swysoski\} @aut.ac.nz
}

\begin{abstract}
The paper introduces a novel connectionist approach to neural network modelling that integrates dynamic gene networks within neurons with a neural network model. Interaction of genes in neurons affects the dynamics of the whole neural network. Through tuning the gene interaction network and the initial gene/protein expression values, different states of the neural network operation can be achieved. A generic computational neurogenetic model is introduced that implements this approach. It is illustrated by means of a simple neurogenetic model of a spiking neural network (SNN). Functioning of the SNN can be evaluated for instance by the field potentials, thus making it possible to attempt modelling the role of genes in different brain states such as epilepsy, schizophrenia, and other states, where EEG data is available to test the model predictions.
\end{abstract}

\section{INTRODUCTION}

The genes, encoded in the DNA, that are transcribed into RNA, and then translated into proteins in each cell, contain important information related to the brain activity. A specific gene from the genome relates to the activity of a neuronal cell in terms of a specific function. But the functioning of the brain is much more complex than that. The interaction between the genes is what defines the functioning of a neuron. Even in the presence of a mutated gene in the genome that is known to cause a brain disease, the neurons can still function normally provided a certain pattern of interaction between the genes is maintained [1]. On the other hand, if there is no mutated gene in the genome, certain abnormalities in the brain functioning can be observed as defined by a certain state of the interaction between the genes [2]. The above cited and many other observations point to the significance of modelling a neuron and a neuronal ensemble at the gene level in order to predict the state of the ensemble. The process of modelling the gene interaction for the purpose of brain understanding is a significant challenge to scientists. In [3] methods for evolving connectionist systems are presented where the neural network structure and functionality evolve over time from incoming data, depending on the values of single "gene" parameters. In [4] neural networks are related not to single genes but to gene networks (GN). In [5] a gene interaction network is used in a neural development model of Drosophila.

This paper further develops the evolving connectionist system theory. Some main principles of computational neurogenetic modelling are presented in $[6,7]$. Here we introduce a generic computational neurogenetic model that is illustrated by means of a simple neurogenetic model of a spiking neural network. Results of preliminary simulations are presented.

\section{GENE NETWORKS IN REAL CELLS (NEURONS)}

In a single cell, the DNA, RNA and protein molecules interact continuously during the process of the RNA transcription from DNA (genotype), and the subsequent RNA to protein (phenotype) translation $[8,9]$. A single gene interacts with many other genes in this process, inhibiting, directly or indirectly, the expression of some of them, and promoting others at the same time. This interaction can be represented as a gene regulatory network (GRN). GRN dynamically evolve and change their structure based on DNA and environmental information. Modelling GRN is an extremely difficult task that requires a large amount of data and sophisticated information methods. A large amount of data on gene interactions for specific genomes, as well as on partial models, is available from public domain databases such as NCBI, KEGG, Stanford Microarray Database, and many more. Collecting both static and time course gene expression data from up to 30,000 genes is now a common practice in many biological, medical and pharmaceutical laboratories in the world through the introduction of microarray technologies (see for example http://www.ebi.ac.uk/microarray).

In the gene network (GN), the activation of each gene is a complex function of the activation of other genes in the cell. The GN actually used in our simulations is illustrated in figure 1 . Activations of all gene nodes in a GN at a time moment represent the gene state of the GN. Depending on the initial state of a GN the time development of the GN may follow a different trajectory in the state space as it is illustrated in figure 2 . 


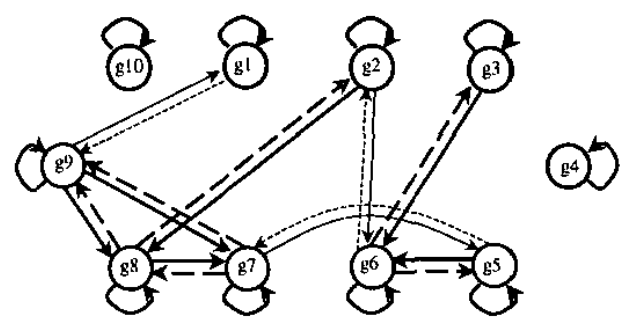

Fig. 1. Optimised gene network (GN) of genes from Table I. Solid (dashed) line means the interactiòn strength $>0(<0)$, respectively. Only connections with $0.6 \geq\left|w_{i j}\right| \geq 0.25$ are illustrated, all other connections are weaker.

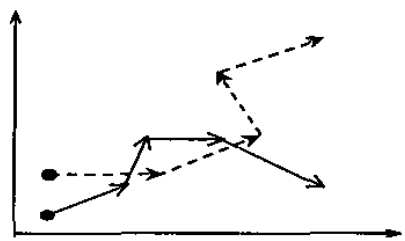

Fig.2. In a hypothetical state space two trajectories represent the consecutive states of a GN when two different initial gene states were chosen (for example: in one of them some gene(s) may be mutated; or different conditions may apply - e.g. treatment or no treatment of a disease).

The brain consists of many interrelated neural networks (NN). Each of them is a tremendously complex adaptive information system characterised by learning, generalisation and development. Every neuron contains the whole genome of the organism and therefore its functions are defined by both the environment it learns from and by the GN that is activated in this neuron. Adaptation of the GN now takes place along with the adaptation process of the NN that makes the modelling of real NNs complex.

A generic computational neurogenetic model that has a GN within a NN is presented in the next section.

\section{A GENERIC COMPUTATIONAL NEUROGENETIC (CNG) MODEL}

In general, we consider two sets of essential genes - a set $\mathbf{G}_{\text {gen }}$ that defines generic neuronal functions (e.g., general cell life functions) and a set $\mathbf{G}_{\text {spec }}$ that defines specific neuronal functions (e.g. receptors, ion channels, etc.). The two sets form together a set $\mathbf{G}=\left\{G_{l}, G_{2}, \ldots, G_{n}\right\}$. We do not know the absolute values of gene expression levels therefore we work with the relative changes in their expression. A change in expression level of each gene $g_{j}(t+\Delta t) \in(-\infty, \infty)$ is a function of the changes in gene expression levels of the rest of the genes in $\mathbf{G}(t)$. As the first simple model, we will assume this function to be a linear function, i.e.:

$$
g_{j}(t+\Delta t)=\sum_{k=1}^{n} w_{j k} g_{k}(t)
$$

The square matrix of gene connection weights $\mathbf{W}$ represents the $\mathrm{GN}, w_{i j} \in(-1,1)$ (see figure 1). We assume that when a gene is upregulated (i.e., $g_{j}(t)>0$ ) more protein defined by this gene will be produced in the neuron, and vice versa, i.e. when the gene is downregulated (i.e., $\left.g_{j}(t)<0\right)$ less protein coded by this gene will be produced.

Neuronal functions (neuron's parameters) $\mathbf{P}=\left\{P_{l}\right.$, $\left.P_{2}, \ldots, P_{\mathrm{m}}\right\}$ from a neural network model are related to particular proteins, so that each parameter $P_{j}$ is a function of the expression of several (or in partial case - one) genes. For simplicity in our model we will assume that one parameter $P_{j}$ depends only on one gene such that:

$$
P_{j}(t+\Delta t)=P_{j}(0) g_{j}^{\prime}(t+\Delta t)
$$

where $g_{j}^{\prime}(t+\Delta t) \in[0, \infty)$ is a relative change in the protein concentration against its initial concentration at time 0 , based on the change in its gene expression level. It is calculated as

$$
g_{j}^{\prime}(t)=s(g(t))
$$

where $s$ is a squashing function (see e.g. figure 3 ) and $g(t)$ is determined by equation (1).

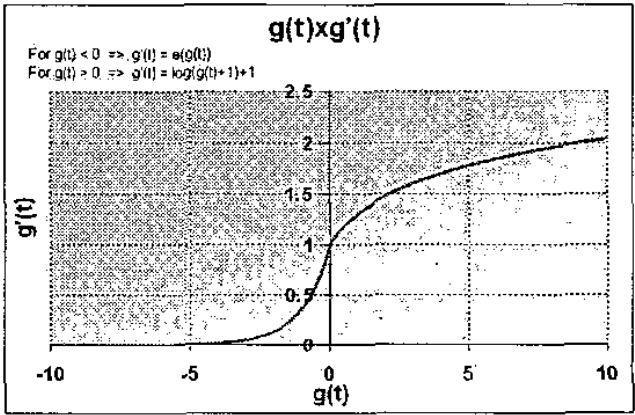

Fig. 3. An example of a nonlinear function to obtain relative changes in parameters values (equation (3)) according to the current changes in gene expression levels $\mathbf{g}(\mathrm{t})$

This generic CNG model can be run step by step over time in the following way:

1) Define the initial changes in expression values of the genes in the neuron, $\mathbf{g}(t=0)$, and the matrix $\mathbf{W}$ of the GN if that is possible. Set the initial values of SNN parameters, $\mathbf{P}(t=0)$.

2) Update the GN and define the next state of the gene vector $\mathbf{g}(t+\Delta t)$ using equation (1).

3) Derive the values of the parameters $\mathbf{P}$ from the gene state $g(t+\Delta t)$ using equation (2).

4) Evaluate the spiking activity of neuron(s) (taking into account all external inputs to the neural network).

5) Go to step 2 . 
We are making several simplifying assumptions:

1. Each neuron has the same GN - in terms of same genes and same network matrix $\mathbf{W}$.

2. Each GN starts from the same initial value of the gene expressions.

3. Each individual GN is synchronized with others, i.e. $\Delta t$ is equal for all genes and for all GNs.

4. There is no feedback from neuronal activity to gene expression level.

Later in our research, these simplifying assumptions can be relaxed.

\section{A. Determination of the GN transition matrix $\mathbf{W}$}

The biggest challenge of our approach and the key to the predictions of our model is the construction of the GN transition matrix $\mathbf{W}$, which determines the dynamics of GN and consequently the dynamics of the NN. There are several ways to obtain $\mathbf{W}$ :

(1) Ideally, the values of gene interaction coefficients $w_{i j}$ are obtained from real measurements through reverse engineering performed on the microarray data [10]. At present, there are very limited experimental data available for the brain.

(2) The values of $\mathbf{W}$ elements are iteratively optimised from initial random values, for instance with the use of genetic algorithms, to obtain the desired behaviour of the NN. The desired behaviour of the NN can simulate certain brain states like epilepsy, schizophrenic hypofrontality, learning and memory disorders (after incorporation of synaptic learning [11]), etc. This behaviour would be used as a "fitness criterion" in the genetic algorithm to stop the search process for an optimal interaction matrix $\mathbf{W}$.

(3) The matrix $\mathbf{W}$ is constructed heuristically based on some assumptions and insights into what result we want to obtain and why. For instance, we can use the theory of discrete dynamic systems to obtain a dynamic system with the fixed point attractor(s), limit cycle attractors or strange attractors [12].

(4) The matrix $W$ is constructed from known facts and literature on gene-protein interaction.

(5) The matrix $\mathbf{W}$ is constructed with the use of a mix of the above methods.

\section{B. Model defined GN corresponding to the desired NN behaviour}

The above generic model allows us to investigate and discover relationships between different GNs and NN states. A procedure to obtain this relationship can read:

1. For an initial GN state, generate a GN matrix $\mathbf{W}$;

2. For the matrix $\mathbf{W}$ run the $\mathrm{NN}$ model over a period of time $\mathrm{T}$ and record the activation of the neurons in the NN;
3. Evaluate characteristics of the $\mathrm{NN}$ behaviour (e.g. total activation, spectral characteristics, etc);

4. Compare the NN characteristics to the characteristics of the desired NN state (e.g. epilepsy);

5. Repeat steps (1) to (4) until a desired GN and NN model behaviour is obtained;

6. Analyse the GN and the NN parameters for significant gene patterns that cause the NN model behaviour.

The generic model above is illustrated in the next sections on a simple CNG model of a spiking neural network (SNN) with an optimised GN.

\section{A CNG MODEL OF A SPIKING NEURAL NETWORK (SNN) - MODEL DESCRIPTION}

We design a small network of spiking neurons, $\mathrm{N}=120$. Inside of each neuron a small gene network $(\mathrm{GN})$ affects the values of neuron parameters in a dynamic fashion (figure 4). Each neuron parameter is in reality linked to a particular protein (receptor, ion channel, enzyme, etc.) the concentration of which is determined by the expression level of the corresponding gene(s).

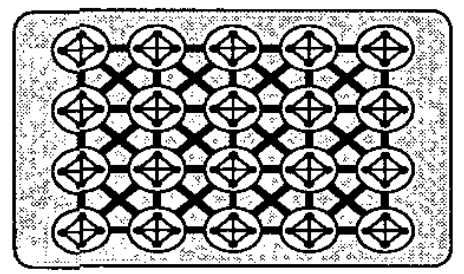

Fig. 4. Neurons of the model neural network have a gene network operating within them that affects the values of their parameters in a dynamic fashion.

Our neuron spiking model is derived from the spike response model (SRM) [13]. The total somatic postsynaptic potential (PSP) of a neuron $i$ is $u_{i}(t)$. When $u_{i}(t)$ reaches the firing threshold $\vartheta_{i}(t)$, neuron $i$ fires, i.e. emits a spike (see figure 5). The moment of $\vartheta_{i}(t)$ crossing defines the firing time $t_{i}$ of an output spike. The value of $u_{i}(t)$ is the weighted sum of all synaptic PSPs, $\varepsilon_{i j}\left(t-t_{j}-\Delta_{i j}^{a x}\right)$, such that:

$$
u_{i}(t)=\sum_{j \in \Gamma_{i} j_{j} \in F_{j}} J_{i j} \varepsilon_{i j}\left(t-t_{j}-\Delta_{i j}^{a r}\right)
$$

The weight of synaptic connection from neuron $j$ to neuron $i$ is denoted by $J_{i j}$. It takes positive (negative) values for excitatory (inhibitory) connections, respectively. $\Delta_{i j}^{a x}$ is an axonal delay between neurons $i$ and $j$, which linearly increases with Euclidean distance between neurons. The positive kemel expressing an individual postsynaptic potential (PSP) evoked on neuron $i$ when a presynaptic 
neuron $j$ from the pool $\Gamma_{\mathrm{i}}$ fires at time $t_{j}$ has a double exponential form, i.e.

$$
\varepsilon_{i j}^{\text {syapse }}(s)=A^{\text {synapse }}\left(\exp \left(-\frac{s}{\tau_{\text {decay }}^{\text {Nmapse }}}\right)-\exp \left(-\frac{s}{\tau_{\text {rise }}^{\text {synapse }}}\right)\right)
$$

where $\tau_{\text {decay/rise }}^{\text {synase }}$ are time constants of the fall and rise of an individual PSP, respectively, $A$ is the PSP's amplitude, and synapse denotes one of the following: fast_excitation, fast_inhibition, slow_excitation, and slow_inhibition. These types of PSPs are based on neurobiological data [14]. Immediately after firing the output spike at $t_{i}$, neuron's firing threshold $\vartheta_{i}(t)$ increases $k$-times and then returns to its resting value $\vartheta_{0}$ in an exponential fashion:

$$
\vartheta_{i}\left(t-t_{i}\right)=k \times \vartheta_{0} \exp \left(-\frac{t-t_{i}}{\tau_{\text {decay }}^{\delta}}\right)
$$

where $\tau_{\text {decay }}^{\theta}$ is the time constant of the threshold decay. In such a way, absolute and relative refractory periods are modelled. External inputs from the input layer are added to the right hand side of (4) at each time step. Each external input has its own weight $J_{i}^{\text {ext } \text { Input }_{\text {ind }} \text { and } \varepsilon_{i}^{\text {fast }} \text { excication }}(t)$, i.e.

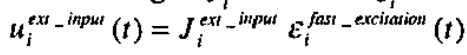

We employed a random input with the average fring frequency of $15 \mathrm{~Hz}$.

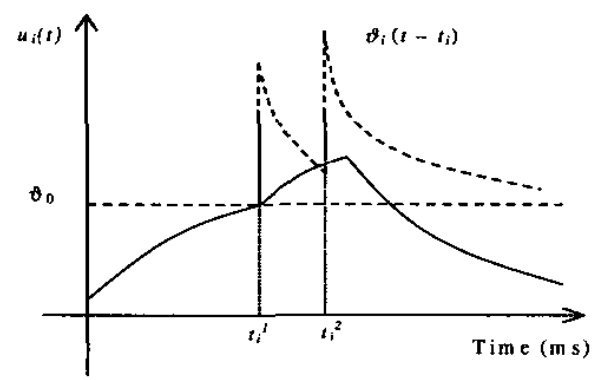

Fig. 5. Spiking neuron model. When the state variable $u_{i}(t)$ of a spiking neuron reaches the firing threshold $\vartheta_{i}(t)$ at time $t_{i}$, the neuron fires an output spike. Current firing threshold rises after each output spike and decays back to the initial value.

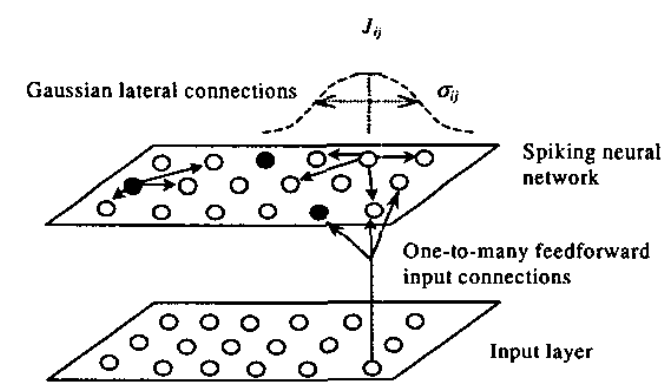

Fig. 6. SNN architecture. Filled circles denote inhibitory neurons.
Figure 6 illustrates the architecture of our spiking neural network (SNN). Spiking neurons within the network can be either excitatory or inhibitory. There can be as many as about $10-20 \%$ of inhibitory neurons positioned randomly on the rectangular grid of $N$ neurons. Lateral connections between neurons have weights that decrease in value with distance from neuron $i$ according to a Gaussian formula while the connections between neurons themselves can be established at random. There are one-to-many feed-forward connections from the input layer decreasing in strength according to the Gaussian distribution. Table I and II contain the values of neuron's and SNN parameters, respectively, that were used in our preliminary simulations.

TABLE I

NEURON'S PARAMETERS

\begin{tabular}{||c|l|c|}
\hline \hline GENE & NEURON'S PARAMETER & VALUE \\
\hline G1 & Amplitude of fast excitation & 4 \\
\hline G2 & Fast excitation rise / decay time constants (ms) & $2 / 5$ \\
\hline G3 & Amplitude of slow excitation & 1 \\
\hline G4 & Slow excitation rise / decay time constants (ms) & $20 / 50$ \\
\hline G5 & Amplitude of fast inhibition & 1 \\
\hline G6 & Fast inhibition rise / decay time constants (ms) & $5 / 10$ \\
\hline G7 & Amplitude of slow inhibition & 3 \\
\hline G8 & Slow inhibition rise / decay time constants (ms) & $50 / 100$ \\
\hline G9 & Resting firing threshold & 19.5 \\
\hline G10 & Decay time constant of the firing threshold (ms) & 30 \\
\hline- & Number of times the threshold is increased $k$ & 2 \\
\hline
\end{tabular}

TABLE II

SNN PARAMEters

\begin{tabular}{||l|c|}
\hline \hline SNN PARAMETER & VALUE \\
\hline Number of neurons & 120 \\
\hline Proportion of inhibitory neurons & 0.2 \\
\hline Probability of external input fiber firing & 0.015 \\
\hline Peak/sigma of extemal input weight & $5 / 1$ \\
\hline Peak/sigma of lateral excitatory weights & $10 / 4$ \\
\hline Peak/sigma of lateral inhibitory weights & $40 / 6$ \\
\hline Probability of connection & 0.5 \\
\hline
\end{tabular}

Table I also contains relations between neural parameters and hypothetical genes in our GN. For instance, amplitudes of PSPs would be related to the concentration of receptorgated ion channels in the postsynaptic membrane. The time constants of PSPs would be related to the properties of individual receptor-gated ion channels. Concentrations and properties of proteins are determined by coding genes.

We keep record of spiking activities of all neurons individually and in total, as well as the record of the field potential that is in the brain proportional to EEG [15]. Activity changes will be related to the evolution of gene expression in neurons. For demonstration of the behaviour of our model we use the average of all instantaneous membrane potentials, thus obtaining the network field 
potential $\Phi(\mathrm{t})=(1 / \mathrm{N}) \sum u_{i}(t)$. For its analysis we use the fast Fourier method [16].

\section{SOME PRELIMINARY EXPERIMENTAL RESULTS}

Figure 7 illustrates the field potential of our SNN model and its spectral characteristics for an optimised GN matrix. $\mathbf{W}$ has been optimised to yield the spectral characteristics of $\Phi$ as similar as possible to the spectral characteristics of normal state EEG [17]. We generated the coefficients of $\mathbf{W}, w_{i j} \in(-1,1)$, such that the modulus of the maximal (complex) eigenvalue was equal to 1 . That means the stable oscillatory behaviour in the Lyapunov sense of the corresponding linear gene dynamic system (see figure $8 \mathrm{c}$ ). The initial values of parameters are listed in Tables I and II. Parameter changes were applied after each $1000 \mathrm{~ms}$ of SNN simulation according to the equation (2).

(a)
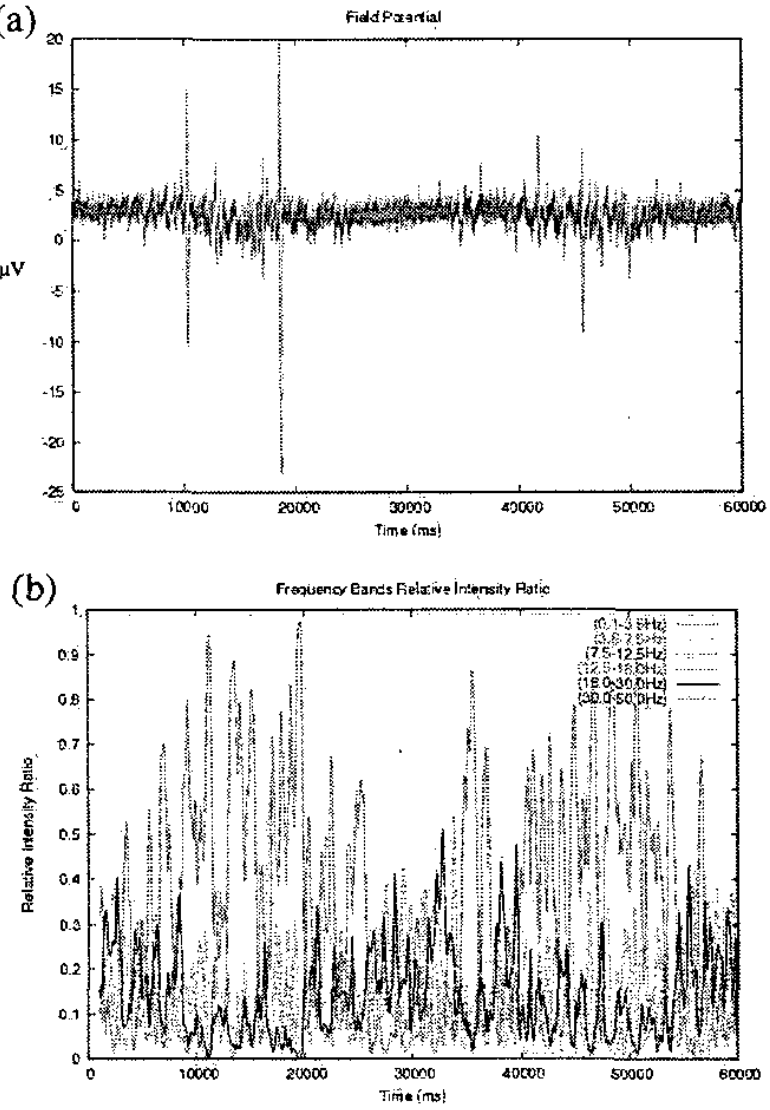

Fig. 7. Time evolution of the field potential of the SNN with dynamic parameter values. (a) Field potential. (b) Spectral characterisation Sampling rate $=1000 \mathrm{~Hz}, \mathrm{Min} / \mathrm{Max}$ frequency $=0.1 / 50 \mathrm{~Hz}$, respectively The dominant frequency band is delta $(0.1-3.5 \mathrm{~Hz})$.
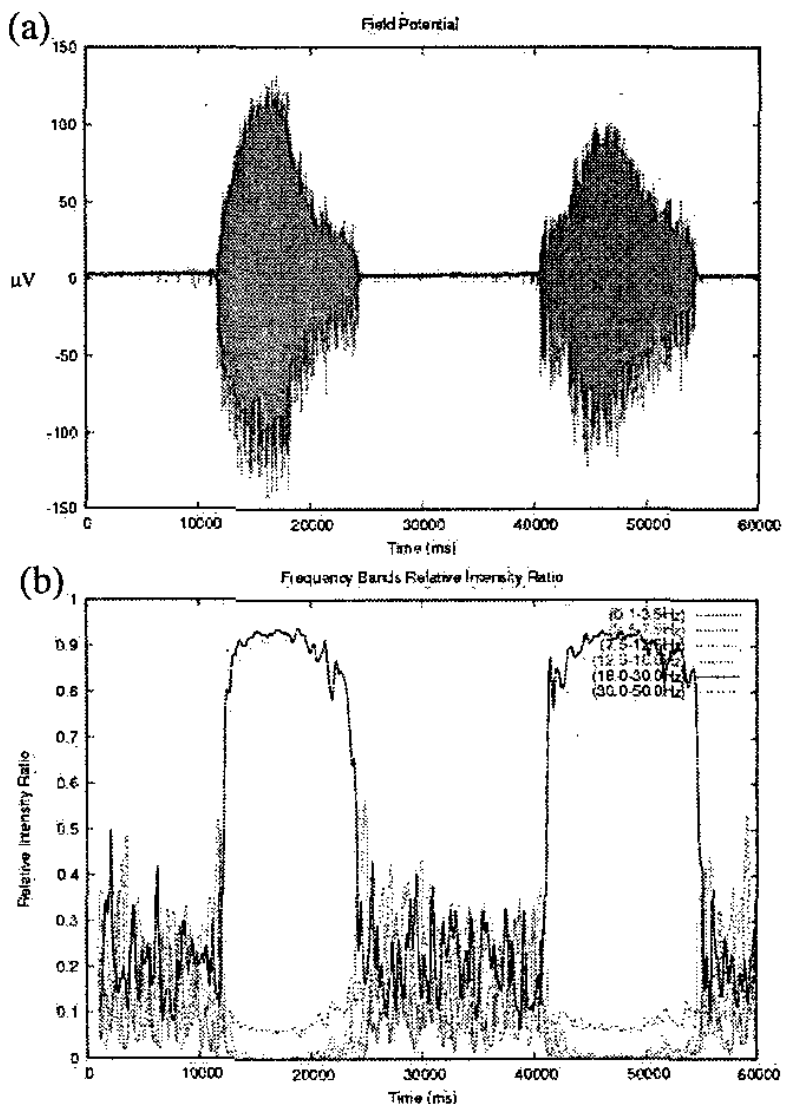

(c)

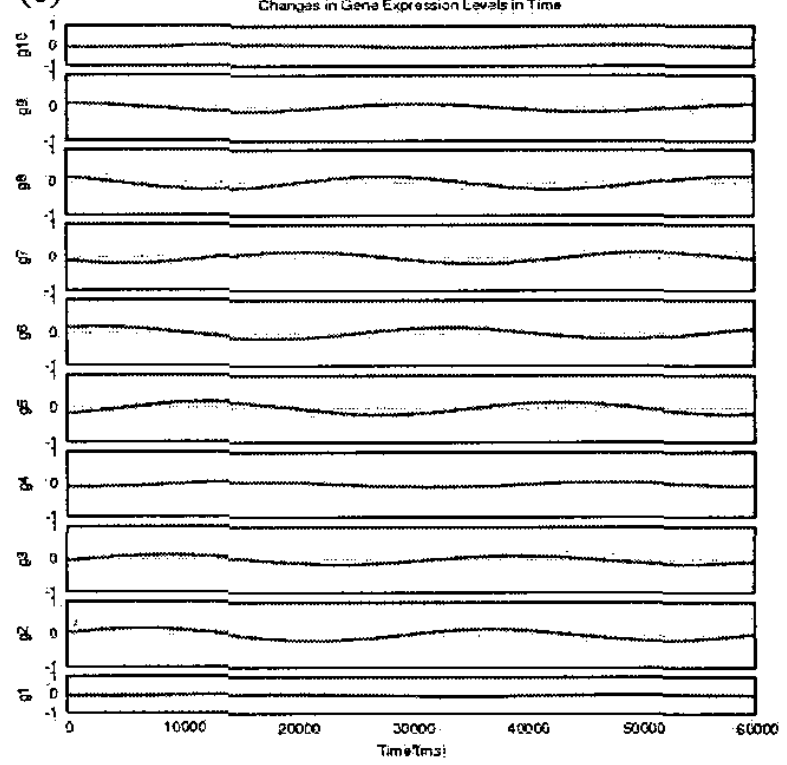

Fig. 8. (a, b) Different initial values of parameters can lead to totally different behaviour of SNN. (c) Corresponding relative changes in gene expression levels for a single optimised interaction matrix $\mathbf{W}$, the same as in figure 7. Zero means no change in the expression level. 
Then we kept the same $\mathbf{W}$ optimised for the normal state EEG and experimented with different initial values of parameters $P_{j}(0)$ in equation (2). Figure 8 illustrates the field potential $\Phi$ of the SNN with initial conditions simulating the temporal lobe epilepsy (TLE) with tonicclonic (TC) seizures [18]. Namely, the slow inhibition was disabled by putting $A^{\text {slow_intibition }}=0$ and the fast inhibition was enhanced by putting $A^{\text {fast_intibition }}=10$. In figure $8 \mathrm{a}, \mathrm{b}$ we can see that the behaviour of the network has completely changed. Now, the neural dynamics leads to transient global synchronizations with frequency characteristics very similar to the EEG of TC seizures for which the dominant frequency is beta $2(18-30 \mathrm{~Hz})$ and the EEG amplitude rises to hundreds of $\mu \mathrm{V}$ [17]. It is worth to mention that with the fixed values of parameters, it is not possible to obtain transitions from the normal state to the prolonged periods of global synchronizations and back as seen in figure 8a. It seems that the internal dynamics of genes linked to neural parameters is needed to account for observed transitions in neural network states. Analysis of which genes and which parameters are crucial for these state transitions can bring new insights into the etiopathogenesis and treatment of the disease.

\section{DISCUSSION}

In real neural networks neurons' parameters that define the functioning of a neural network depend on genes and proteins in a complex way. Gene and protein expression values may also change due to internal dynamics of the gene regulatory (interaction) network, initial conditions of the genes and external conditions. All this may affect gradually or quickly the functioning of the neural network as a whole. It is observed for example that different initial gene conditions can lead to the same outcome in terms of neuronal activity. On the other hand, in the diseased brain, either altered initial conditions, mutated genes and/or altered interactions within GN lead to abnormalities in network activity. Realistic models of gene networks within neural networks should account for these processes.

In order to investigate these phenomena, we have set up a novel model of a SNN that is simple and biologically plausible. It uses principles from the simple spiking neuron models [13]. More detailed models of SNN include for instance detailed ion receptor and channel kinetics [14] and also multiple neuron compartments [19]. It may prove necessary to include also subcortical areas in our CNG model [14]. Further investigation of our model will be performed along with more experiments on real data for model verification before the model is used to explain and predict epileptic, schizophrenic or other neural data.

\section{ACKNOWLEDGMENT}

The research is funded by the Knowledge Engineering and Discovery Research Institute, Auckland University of Technology, Auckland, New Zealand (www.kedri.info).

\section{REFERENCES}

[1] R. Morita, E. Miyazaki, C. G. Fong, X.-N. Chen, J. R. Korenberg, A. $V$. Delgado-Escueta and K. Yamakawa, "JH8, A gene highly homologous to the mouse jerky gene, maps to the region for childhood absence epilepsy on 8q24," Biochem. Biophys. Res. Communications, vol. 248, pp. 307-314, 1998.

[2] V. Crunelli and N. Leresche, "Childhood absence epilepsy: genes, channels, neurons and networks," Nature Rev. Neurosci, vol. 3, pp. $371-382,2002$.

[3] N. Kasabov, "Evolving connectionist systems for adaptive learning and knowledge discovery: methods, tools, applications," Proceedings of the. First International IEEE Symposium on Intelligent Systems, 2002, vol. 1, pp. 24-28.

[4] N. Kasabov, Evolving Connectionist Systems. Methods and Applications in Bioinformatics, Brain Study and Intelligent Machines, London: Springer-Verlag, 2003.

[5] G. Mamellos and E. D. Mjolsness, "Gene network models and neural development," in Modeling Neural Development, A. vanOoyen, Cambridge, MA: MIT Press, 2003, pp. 27-48.

[6] N. Kasabov and L. Benuskova, "Computational neurogenetics," Journal of Computational and Theoretical Nanoscience, vol. 1, pp. 47-61, 2004.

[7] N. Kasabov and L. Benuskova, "Neuro-, genetic-, and neurogenetic information processing," in Handbook of Computational and Theoretical Nanotechnology, M. Rieth and W.Schommers, Los Angeles: American Scientific Publishers, 2004, in press.

[8] P. Baldi and S. Brunak, Bioinformatics. A Machine Learning Approach, 2nd ed, Cambridge, MA: MIT Press, 2001.

[9] J. Bower and H. Bolouri, eds, Computational Modelling of Generic and Biochemical Networks, Cambridge, MA: The MIT Press, 2001.

[10]N. Kasabov and D. Dimitrov, "A method for gene regulatory network modelling with the use of evolving connectionist systems," Proceedings of the ICONIP'2002 - International Conference on Neuro-Information Processing. Singapore, 2002.

[11] L. Benuskova, V. Rema, M. Ammstrong-James, and F. F. Ebner, "Theory for normal and impaired experience-dependent plasticity in neocortex of adult rats," Proc. Natl. Acad. Sci. USA, vol. 98, pp. 2797-2802, 2001.

[12] A. Katok and B. Hasselblat, Introduction to the Modern Theory of Dynamical Systems, Cambridge, MA: Cambridge Univ. Press, 1995.

[13] W. Gerstner and W. M. Kistler, Spiking Neuron Models, Cambridge, MA: Cambridge Univ. Press, 2002.

[14]A. Destexhe, "Spike-and-wave oscillations based on the properties of GABA $_{\mathrm{B}}$ receptors," J. Neurosci., vol. 18, pp. 9099-9111, 1998.

[15] J. W. Freeman, Mass action in the nervous system, New York: Academic Press, 1975.

[16]R. Q. Quiroga, Quantitative Analysis of EEG Signals: TimeFrequency Methods and Chaos Theory. PhD Thesis, Institute of Physiology and Institute of Signal Processing. Medical University Lübeck: Lüubeck, 1998.

[17]R. Q. Quiroga, Dataset \# 3: Tonic-clonic (Grand Mal) seizures, http://www: vis.caltech.edu/ rodri/data.htm.

[18] R. Cossart, C. Dinocourt, J. C. Hirsch, A. Merchan-Perez, J. DeFelipe, Y. Ben-Ari, M. Esclapez and C. Bemard, "Dendritic but not somatic inhibition is decreased in experimental epilepsy," Nature Neuroscience, vol. 4, pp. 52-62, 2001.

[19] K.-H. Yang, P. J. Franaszczuk and G. K. Bergey, "The influences of somatic and dendritic inhibition on bursting patterns in a neuronal circuit model," Biol. Cybernetics, vol. 89, pp. 242-253, 2003. 